\title{
A new weapon against an old target
}

\author{
Christoph Fiehn* \\ See related research by Lu et al., http://arthritis-research.com/content/13/2/R56
}

\begin{abstract}
High expression of folate receptors is characteristic for the effector cell population of synovial macrophages in synovial inflammation. A new drug conjugate, EC0746, targets the folate inhibitor aminopterin to folate receptors. In vitro studies show that this conjugate acts antiproliferatively and inhibits cytokine production by macrophage cell lines. Moreover, it shows strong anti-arthritic effects in the rat model of adjuvantinduced arthritis in vivo. Toxicity of aminopterin was reduced 40 -fold by using equimolar doses of the drug conjugate. In conclusion, this new treatment approach has the potential to further improve the most successful principle of folate inhibition in the treatment of arthritis.
\end{abstract}

Methotrexate (MTX) has been established as the anchor drug in the treatment of rheumatoid arthritis (RA) for decades. Its major role as an effective and well-tolerated substance impressively demonstrates that the principle of folate inhibition has key anti-inflammatory effects in the pathomechanism of arthritis. MTX enters the cell primarily by two ways: the reduced folate carrier (RFC) and the folate receptor (FR)- $\beta$. The latter is the target of a novel, interesting approach for treating arthritis that was introduced in a previous issue of Arthritis Research \& Therapy [1]. RFC is a transmembrane folate transport mechanism that has a ubiquitous distribution throughout the body $[2,3]$. The high affinity of MTX for RFC may explain why MTX has effects on a large number of cell types. Some are therapeutic targets, such as synovial lymphocytes, but others, such as organ cells of liver or kidney, are sensitive to toxic effects of MTX, thus constituting a dose-limiting factor. In contrast to RFC, FR- $\beta$ has a restricted distribution, mainly on activated myelomonocytic cells and neutrophils [4]. In synovial tissue of

*Correspondence: c.fiehn@acura-kliniken.com

ACURA Centre for Rheumatic Diseases, Rotenbachtalstr. 5, 76530 Baden-Baden, Germany
RA patients it has been shown that FR- $\beta$ is selectively expressed on activated monocytes and synovial macrophages and that MTX can enter the cell through receptormediated endocytosis [5]. The monocyte/macrophage population of the inflamed synovia is a key effector cell of inflammation and main source of cytokines such as TNFalpha [6]. MTX may therefore mediate important antiinflammatory effects through its effect on synovial macrophages in RA. Moreover, reduction of cardiovascular mortality of RA patients by MTX is also thought to be mediated by an effect on this cell lineage, as it has been shown that MTX reduces foam cell formation by lipid-laden macrophages [7].

The specific expression of folate receptors in synovial tissue of RA patients has been used to develop methods to image activated macrophages in the rat model of adjuvant-induced arthritis [8]. Moreover, a recombinant variable-region antibody fragment $(\mathrm{Fv})$ against $\mathrm{FR}-\beta$, which was coupled to Pseudomonas exotoxin A (PE38), was shown to inhibit RA synovial macrophages in vitro and has strong anti-inflammatory effects in a human SCID mouse model for RA in vivo [9]. Furthermore, efforts have been taken to identify FR- $\beta$-specific folate inhibitors, which allow specific targeting of the FR- $\beta$ expressing cells with no affinity to RFC [10].

Targeting folate receptors is therefore a way of focusing the effector cell population of synovial macrophages and thus has potential as a specific treatment of synovial inflammation. Lu and colleagues in this issue [1] present a novel FR-specific agent that has the potential to bring this approach much closer to clinical use. They use a novel construct, EC0746, which consists of a folate moiety and the molecule aminopterin (AMT), both connected by a saccharo-amino acid peptide spacer and a hydrazide/disulfide linker. AMT is a folate antagonist and closely related to MTX. While it has strong anti-folate effects, its use as a free drug was restricted by frequent toxicity, which is why it did not find its way into clinical practice. The folate moiety of EC0746 binds the conjugate to FR- $\beta$, thus targeting the drug to synovial macrophages in synovial inflammation. While the peptide spacer reduces hepatic clearance during circulation of the drug, the chemical linker is rapidly cleaved in the endosomal structures when the drug conjugate is taken up into the 
cell by receptor-mediated endocytosis. Therefore, EC0746 is a classic example of the application of targeted drug delivery, in this case the targeting of the antifolate (AMT) to FR- $\beta$-carrying cells. Lu and colleagues performed a number of in vitro and in vivo studies that showed the high anti-arthritic potential of this construct. EC0746 has a high binding specificity for FR- $\beta$-expressing cells. It acts antiproliferatively on one FR- $\beta$-expressing macrophage-derived cell line and blocks cytokine production after stimulation with lipopolysaccharide and interferon- $\gamma$ in another. For in vivo studies, the rat model of adjuvant-induced arthritis was used, which is characterized by a high inflammatory response dominated by a strong activation of macrophages. Treatment with EC0746 given subcutaneously twice weekly showed a very good response with about $91 \%$ inhibition of paw edema and effective suppression of the systemic signs of the disease, such as weight loss and splenomegaly. The effect was strongly superior to those of MTX and as well the TNF-inhibitor etanercept. Most interestingly, EC0746 was markedly safer than native AMT, with a 40-fold difference in toxicity, which is explained by the fact that the conjugate is constructed to be cleaved to the active drug after cellular uptake only.

Treatment with EC0746 is an interesting novel approach that uses an FR-specific construct to target the folate inhibitor AMT to activated synovial macrophages. However, several questions remain: will the folate intake have to be restricted in clinical trials in order to prevent competition for FR- $\beta$ ? Epithelial cells carry FR- $\alpha$, which will bind EC0745 as well. Will this result in toxicity? The development of targeted drug delivery towards folate receptors for clinical use is still in its infancy. Nevertheless, new approaches demonstrate that targeting pathways of folate metabolism is still good for new weapons against arthritis.

\section{Abbreviations}

AMT, aminopterin; FR, folate receptor; MTX, methotrexate; RA, rheumatoid arthritis; RFC, reduced folate carrier; TNF, tumor necrosis factor.
Competing interests

The author declares that he has no competing interests.

Published: 12 August 2011

\section{References}

1. Lu Y, Stinnette TW, Westrick E, Klein PJ, Gehrke MA, Cross VA, Vlahov IR, Low PS, Leamon CP: Treatment of experimental adjuvant arthritis with a novel folate receptor-targeted folic acid-aminopterin conjugate. Arthritis Res Ther 2011, 13:R56.

2. Fiehn C: Methotrexate transport mechanisms: the basis for targeted drug delivery and $\beta$-folate-receptor-specific treatment. Clin Exp Rheumatol 2010, 28(5 Suppl 61):S40-45

3. Kremer JM: Toward a better understanding of methotrexate. Arthritis Rheum 2004, 50:1370-1382.

4. Elnakat $H$, Ratnam M: Distribution, functionality and gene regulation of folate receptor isoforms: implications in targeted therapy. Adv Drug Deliv Rev 2004, 56:1067-1084

5. Nakashima-Matsushita N, Homma T, Yu S, Matsuda T, Sunahara N, Nakamura T, Tsukano M, Ratnam M, Matsuyama T: Selective expression of folate receptor beta and its possible role in methotrexate transport in synovial macrophages from patients with rheumatoid arthritis. Arthritis Rheum 1999, 42:1609-1616.

6. Feldmann M, Brennan FM, Maini R: Role of cytokines in rheumatoid arthritis. Annu Rev Immuno/ 1996, 14:397-440

7. Reiss AB, Carsons SE, Anwar K, Rao S, Edelman SD, Zhang H, Fernandez P, Cronstein BN, Chan ES: Atheroprotective effects of methotrexate on reverse cholesterol transport proteins and foam cell transformation in human THP-1 monocyte/macrophages. Arthritis Rheum 2008, 58:3675-3683.

8. Turk MJ, Breur GJ, Widmer WR, Paulos CM, Xu LC, Grote LA, Low PS: Folatetargeted imaging of activated macrophages in rats with adjuvant-induced arthritis. Arthritis Rheum 2002, 46:1947-1955.

9. Nagayoshi R, Nagai T, Matsushita K, Sato K, Sunahara N, Matsuda T, Nakamura T, Komiya S, Onda M, Matsuyama T: Effectiveness of anti-folate receptor beta antibody conjugated with truncated Pseudomonas exotoxin in the targeting of rheumatoid arthritis synovial macrophages. Arthritis Rheum 2005, 52:2666-2675.

10. van der Heijden JW, Oerlemans R, Dijkmans BA, Qi H, van der Laken CJ, Lems WF, Jackman AL, Kraan MC, Tak PP, Ratnam M, Jansen G: Folate receptor beta as a potential delivery route for novel folate antagonists to macrophages in the synovial tissue of rheumatoid arthritis patients. Arthritis Rheum 2009 60:12-21.

doi:10.1186/ar3392

Cite this article as: Fiehn C: A new weapon against an old target. Arthritis Research \& Therapy 2011, 13:122. 